\section{Neumonía neumocócica bacteriémica en adultos en hospital regional de Chile}

\author{
MARÍA LUISA RIOSECO Z., RAÚL RIQUELME O., \\ MAURICIO RIQUELME O., CARLOS INZUNZA P., \\ JAVIER RIQUELME D., ANDREA SANHUEZA R.
}

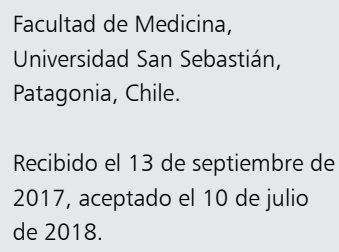

Los autores declaran no tener conflictos de interés.

Trabajo no recibió

financiamiento.

Correspondencia a: María Luisa Rioseco Z. malurioseco@gmail.com

Background: Bacteremic pneumococcal pneumonia (BPP) is a preventable disease with high morbimortality. Aim: To evaluate clinical aspects and mortality on BPP patients admitted to a Chilean regional hospital. Patients and Methods: We looked for adult patients with Streptococcus pneumoniae isolated from blood cultures between 2010 and 2014 years and reviewed clinical records of those who were admitted with pneumonia. Results: We identified 70 BPP patients: $58 \%$ were men, mean age was 56 years, 30\% were $>65$ years, $70 \%$ with basic public health insurance, $26 \%$ were alcoholics, $86 \%$ had comorbidities. Only two patients were vaccinated against $S$. pneumoniae. CURB-65 severity index for community acquired pneumonia was $>3$ in $37 \%$ of patients. Twenty-four patients were admitted to ICU, twenty required mechanical ventilation and twenty-four died (34\%). Mortality was associated with an age over 65 years, presence of comorbidities and complications of pneumonia. A total of 22 serotypes of $S$. pneumoniae were identified, five of them $(1,3,7 F, 14$ y $9 \mathrm{~V})$ were present in 57\% of cases. Conclusions: Elevated mortality of our BNN patients was associated with comorbidities and possibly with socio economic factors, which conditioned a late access to medical care.

(Rev Med Chile 2018; 146: 839-845)

Key words: Pneumococcal Infections; Pneumonia, Pneumococcal; Serogroup; Streptococcus pneumoniae.

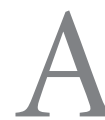
pesar de la disponibilidad de vacunas, Streptococcus pneumoniae continúa siendo un agente relevante en diversos cuadros infecciosos y es el principal microorganismo identificado en las neumonías comunitarias del adulto $^{1-3}$. La frecuencia y letalidad de la enfermedad neumocócica invasiva (ENI) determinaron que en distintos países, entre ellos Chile, se incorporara la vacunación en niños y grupos específicos de adultos. La ENI en adultos se manifiesta principalmente como neumonía bacteriémica afectando especialmente a mayores de 65 años con comorbilidades y con cifras de letalidad que pueden llegar sobre $20 \%{ }^{4-6}$. La bacteriemia ocurriría en aproximadamente $25 \%$ de las neumonías causadas por Streptococcus pneumoniae lo que ha sido generalmente asociado con mayor letalidad ${ }^{7-9}$. Nuestro propósito fue caracterizar los casos de neumonía neumocócica bacteriémica (NNB) ingresados al Hospital Puerto Montt (HPM) entre 2010 y 2014 describiendo aspectos clínicos y factores asociados a mortalidad. 


\section{Pacientes y Método}

Estudio realizado en el HPM, hospital general docente de 440 camas. Se analizaron datos clínicos y epidemiológicos de mayores de 15 años con hemocultivos (HC) positivos para S. pneumoniae, hospitalizados entre enero de 2010 y diciembre de 2014. Se incluyeron sólo casos de neumonía adquirida en la comunidad (NAC) según criterios de Fang ${ }^{10}$.

Del ingreso, se consignaron: fecha, edad, sexo, previsión, uso de antimicrobianos en últimos 15 días, sospecha de aspiración, compromiso de conciencia, consumo de alcohol y tabaco, hospitalizaciones en últimos 90 días, comorbilidades calculando el índice de Charlson ${ }^{11}$, síntomas, signos, exámenes e imágenes radiológicas. El compromiso radiográfico se clasificó como uni o multilobar y se registró la existencia de derrame pleural. Para severidad se utilizó el índice CURB$65^{12}$ y en los ingresados a UCI, el APACHE II ${ }^{13}$. Se consideró complicación la cavitación o empiema, el aumento de infiltrados de $50 \%$ en las primeras $72 \mathrm{~h}$ y la aparición de foco extrapulmonar. Se consignó la presencia de shock, insuficiencia renal aguda (IRA), ingreso a UCI, uso de ventilación mecánica (VM), mortalidad y causas, esquema antimicrobiano, sus modificaciones y motivo de ellas. Sepsis se definió como disfunción orgánica con amenaza vital asociada a $\mathrm{HC}$ positivos. El tratamiento inicial fue considerado adecuado si la cepa era sensible in vitro al menos a uno de los antimicrobianos administrados.

La identificación de S. pneumoniae se realizó considerando la morfología de la colonia y test de optoquina. Se realizó antibiograma en difusión que incluyó epsilometría para penicilina y ceftriaxona según recomendaciones del Clinical Laboratory Standards Institute ${ }^{14}$. Las cepas fueron enviadas al Instituto de Salud Pública (ISP) donde se serotipificaron. Dado que en Chile el Programa Nacional de Inmunizaciones (PNI) incluye la vacuna antineumocócica (VAN) Pneumo 23 (Pn23) en adultos $\geq 65$ años, consideramos ese grupo como objetivo de VAN y para identificar los vacunados, revisamos el Registro Nacional de Inmunizaciones (RNI). Se usaron las siguientes definiciones: alcoholismo: consumo de alcohol $>60$ g día; shock: hipotensión (Presión sistólica $<90 \mathrm{mmHg}$ ) refractaria al aporte de fluidos o que requiere vasopresores o anormalidades de la perfusión tisular evidenciadas por estado mental alterado, oliguria o acidosis láctica; IRA se definió como creatinina plasmática inicial $>1,2 \mathrm{mg} / \mathrm{dL}$ o aumento mayor a $2 \mathrm{mg} / \mathrm{dL}$ en pacientes con insuficiencia renal crónica.

\section{Estadística}

Se usó el paquete estadístico SPSS 19. La variables categóricas fueron descritas como frecuencias y porcentajes y las continuas, por media y desviación estándar (DS). Para la comparación de las variables paramétricas se usó la prueba t-test de Student y para los datos discretos $\chi^{2}$ y el test exacto de Fisher cuando fue necesario. Un valor de $\mathrm{p}<0,05$ se consideró estadísticamente significativo.

\section{Resultados}

Se encontraron 112 pacientes mayores de 15 años con al menos un $\mathrm{HC}$ con $S$. pneumoniae. Obtuvimos la ficha clínica en 88 casos (79\%). Se descartaron 7 casos de meningitis, 6 con peritonitis bacteriana espontánea, un paciente con absceso del psoas y 3 bacteriemias sin foco claro. Se excluyó un caso de neumonía nosocomial. Finalmente, 70 casos cumplieron con los criterios de inclusión.

La edad media fue 56,1 $\pm 17,7$ años, $30 \%>65$ años. El $65 \%$ se presentó en otoño-invierno. El $72 \%$ pertenecía a Fonasa A o B. La Tabla 1 muestra las variables demográficas, comorbilidad, condiciones clínicas y radiológicas. El 85,7\% $(\mathrm{n}=60)$ tenía una o más comorbilidades y el índice de Charlson fue de 3,04 $\pm 3,04$ (0-13,2). La hospitalización media fue de 14,1 $\pm 12,8$ (1-59) días. El $16 \%(\mathrm{n}=11)$ había estado hospitalizado en los últimos 90 días y $6 \%$ había recibido antimicrobianos previamente.

Los síntomas fueron disnea (77\%), tos (73\%), fiebre $(73 \%)$ y expectoración $(67 \%)$. Menos frecuentes fueron dolor torácico $(39 \%)$, calofríos (39\%) y confusión $(24 \%)$. Se obtuvo el nivel de albúmina en 36 pacientes y el promedio fue de 2,8 $\pm 0,7 \mathrm{mg} / \mathrm{dL}(1,5-4,3)$.

El tratamiento inicial incluyó ceftriaxona en 97\%, monoterapia en 41 casos (59\%). La asociación principal fue con una quinolona $(\mathrm{n}=27$, $38,5 \%)$. El esquema fue adecuado en $100 \%$. En $65 \%$ se modificó el antimicrobiano por mejoría clínica $(44,5 \%)$, resultado de HC $(30 \%)$ o deterioro $(26 \%)$; en 8 pacientes se cambió a penicilina 
Tabla 1. Caracterización de $\mathbf{7 0}$ pacientes con neumonía neumocócica bacteriémica, Hospital Puerto Montt 2010-2014

\begin{tabular}{|c|c|c|}
\hline & $\mathbf{n}$ & $\%$ \\
\hline Sexo masculino & 48 & 69 \\
\hline Ancianos (> 65 años) & 21 & 30 \\
\hline Fonasa $\mathrm{A}$ y $\mathrm{B}$ & 50 & 71 \\
\hline Alcoholismo & 18 & 26 \\
\hline Comorbilidad & 60 & 86 \\
\hline Cardiopatía & 14 & 20 \\
\hline Enfermedad pulmonar & 15 & 21 \\
\hline Diabetes & 12 & 17 \\
\hline Enfermedad renal & 10 & 14 \\
\hline Hemodiálisis & 4 & 6 \\
\hline Enfermedad hepática & 8 & 11 \\
\hline Cáncer & 7 & 10 \\
\hline Secuela enfermedad neurológica & 9 & 13 \\
\hline Neutropenia $<1.000$ & 4 & 6 \\
\hline Mesenquimopatía & 3 & 4 \\
\hline Tratamiento esteroidal & 5 & 7 \\
\hline $\mathrm{VIH}$ & 2 & 3 \\
\hline Quimioterapia & 2 & 3 \\
\hline \multicolumn{3}{|l|}{ Radiografía } \\
\hline Infiltrados alveolares & 49 & 70 \\
\hline Compromiso multilobar & 30 & 43 \\
\hline Derrame pleural al ingreso & 11 & 16 \\
\hline \multicolumn{3}{|l|}{ Evolución } \\
\hline Empiema y/o cavitación & 17 & 24 \\
\hline Foco extrapulmonar & 5 & 7 \\
\hline Ingreso a UCl & 24 & 34 \\
\hline Ventilación mecánica & 20 & 29 \\
\hline Mortalidad & 24 & 34 \\
\hline Exámenes al ingreso & & Media \\
\hline Hematocrito & 65 & 34,7 \\
\hline Leucocitos $x \mathrm{~mm}^{3}$ & 67 & 16.047 \\
\hline Plaquetas $\times \mathrm{mm}^{3}$ & 62 & 222.625 \\
\hline Creatinina (mg/dl) & 66 & 1,7 \\
\hline Sodio (mEq/L) & 63 & 134 \\
\hline Albúmina (mg/dl) & 25 & 2,8 \\
\hline PAFI & 32 & 329 \\
\hline
\end{tabular}

con el informe de susceptibilidad. El 100\% de las cepas fue sensible a penicilina y ceftriaxona con CIM entre $0,002-2 \mathrm{ug} / \mathrm{mL}$ y $0,002-1 \mathrm{ug} / \mathrm{mL}$ respectivamente.

En 67 cepas se identificaron 22 serotipos (Tabla $2)$, siendo los más frecuentes $1(\mathrm{n}=9), 3(\mathrm{n}=8)$, $7 \mathrm{~F}(\mathrm{n}=8), 14(\mathrm{n}=7)$ y $9 \mathrm{~V}(\mathrm{n}=6)$, responsables de $54 \%$ de los casos. Los serotipos coincidieron con los de la vacuna Pn 23 en $81 \%$ y con los de la vacuna 13 valente $(13 \mathrm{~V})$ en $76 \%$. En el RNI figuraban 8 pacientes vacunados con Pn23, seis en el período posterior al estudio.

En los ingresados a UCI (36\%), el APACHE fue 24,4 $\pm 8,6$ (9-43). Evolucionó con shock el $45 \%, 40 \%$ con sepsis y $35 \%$ con IRA. Fallecieron 24 pacientes $(34,3 \%)$ con mortalidad atribuible a la neumonía en $83 \%$. La causa de muerte fue principalmente falla respiratoria $(50 \%)$ y shock $(25 \%)$. La mortalidad se asoció significativamente con edad $>65$ años, comorbilidades, Charlson $\geq 1,5$ y extensión y complicaciones de la neumonía. No falleció ningún paciente sin comorbilidad. La albúmina media ( $\mathrm{g} / \mathrm{dL} \pm \mathrm{DS}$ ) al ingreso fue significativamente menor en los fallecidos $(2,4 \pm 0,2$ vs $2,9 \pm 0,1 ; p=0,011)$ al igual que el recuento de plaquetas $(173.180 \pm 112.046$ vs $247.951 \pm$ 114.014, $\mathrm{p}=0,017)$. Otros factores asociados a mortalidad del análisis univariado pueden verse en la Tabla 3.

Falleció $62,5 \%$ de los casos con serotipo 3 (5/8) y no falleció ninguno con serotipo $1(0 / 9)$. Los pacientes con serotipos no contenidos en la vacuna $13 \mathrm{~V}$ tuvieron mayor mortalidad que los con serotipos incluidos (56\% vs 29\%). Ambas diferencias no fueron significativas.

Tabla 2. Distribución de serotipos de 67 cepas de Streptococcus pneumoniae aisladas en neumonía bacteriémica, Hospital Puerto Montt 2010-2014

\begin{tabular}{|cccc|}
\hline Serotipo & $\mathbf{n}$ & $\mathbf{\%}$ & Fallecidos \\
\hline 1 & 9 & 13 & $0 / 9$ \\
\hline 3 & 8 & 12 & $5 / 8$ \\
\hline $7 \mathrm{~F}$ & 8 & 12 & $2 / 8$ \\
14 & 7 & 10 & $3 / 7$ \\
\hline $9 \mathrm{~V}$ & 6 & 9 & $3 / 3$ \\
\hline 4 & 4 & 6 & $0 / 4$ \\
\hline $9 \mathrm{~N}$ & 4 & 6 & $2 / 2$ \\
\hline $6 \mathrm{~B}$ & 3 & 5 & $1 / 3$ \\
\hline $11 \mathrm{~A}$ & 2 & 3 & $2 / 2$ \\
\hline $12 \mathrm{~F}$ & 2 & 3 & $1 / 2$ \\
\hline $19 \mathrm{~F}$ & 2 & 3 & $1 / 2$ \\
\hline $23 \mathrm{~F}$ & 2 & 3 & $0 / 2$ \\
\hline Otros & 10 & 15 & $4 / 10$ \\
\hline
\end{tabular}


Tabla 3. Factores asociados a mortalidad en 70 pacientes con neumonía neumocócica bacteriémica, Hospital Puerto Montt 2010-2014 (Análisis univariado)

\begin{tabular}{|lccc|}
\hline & OR & IC 95\% & p \\
Edad $\geq 65$ años & 4,1 & $1,4-12,1$ & 0,010 \\
Presencia comorbilidad & & & 0,011 \\
\hline Índice de Charlson $\geq 1,5$ & 8,5 & $1,8-40,3$ & 0,002 \\
CURB-65 $\geq 2$ & 8,6 & $2,3-33,6$ & 0,000 \\
\hline Creatinina $\geq 1,2 \mathrm{mg} / \mathrm{dL}$ & 3,5 & $1,3-10,1$ & 0,018 \\
Compromiso bilateral & 4,1 & $1,4-11,8$ & 0,009 \\
Compromiso multilobar & 5,0 & $1,7-15$ & 0,003 \\
\hline Complicación & 4,5 & $1,4-14$ & 0,009 \\
\hline PAFI $\leq 300$ & 11,3 & $1,8-69$ & 0,006 \\
Saturación $\leq$ 89\% al ingreso & 3,7 & $1,1-10,3$ & 0,032 \\
\hline Ingreso a UCl & 4,4 & $1,5-12,8$ & 0,005 \\
\hline Ventilación mecánica & 6,6 & $2,1-20,5$ & 0,001 \\
\hline SDRA & 14,1 & $2,7-73,3$ & 0,000 \\
\hline Sepsis & 11,3 & $3,5-37$ & 0,000 \\
\hline Shock & 26,7 & $6,5-110$ & 0,000 \\
\hline
\end{tabular}

El CURB-65 se pudo calcular en 68 casos. El grupo de menor gravedad (CURB-65 $\leq 2$ ) tuvo mortalidad de $18,6 \%$ (8/43) que aumentó a $60 \%$ $(15 / 25)$ en aquellos con CURB-65 $\geq 3$ (Tabla 4).

\section{Discusión}

Nuestro principal hallazgo es la detección y caracterización de un grupo de pacientes con NNB de características especiales y elevada mortalidad, lo que pudiera estar relacionado con distintos factores.

En Chile no existe vigilancia de la ENI que nos permita comparar con datos nacionales. Datos del Ministerio de Salud ${ }^{15,16}$, muestran una tasa de mortalidad general por neumonía de 24.7/100.000 (2013) y en los $>65$ años, una tasa de 223/100.000 (2011). Si consideramos que $50 \%$ de las neumonías en adultos son neumocócicas ${ }^{1-3}$ podríamos estimar que la mitad de los fallecidos de 2011 fueron por neumonía por $S$. pneumoniae.

La letalidad de $34,3 \%$ en estos pacientes con $\mathrm{NNB}$, fue un hallazgo inesperado. La cifra es superior a lo reportado por la literatura nacional e internacional incluyendo nuestra publicación anterior ${ }^{4,5,7-9}$ lo que podría deberse a diversos factores propios del huésped, del microorganismo causal y derivados de la atención.

Respecto del huésped, nuestra población tiene varios elementos que lo definen como vulnerable: un porcentaje importante es $>65$ años, con comorbilidades expresado en un índice de Charlson promedio de 3,04, 26\% son alcohólicos, la mayoría pertenece a un estrato socioeconómico bajo y presentan bajo nivel de albúmina. Sin embargo, estos aspectos no difieren tanto de otros estudios donde la mortalidad es menor ${ }^{3,4}$.

Si consideramos que la ENI es una entidad inmunoprevenible y que existen vacunas efectivas, debiéramos esperar un resultado clínico diferente según el éxito de los programas de vacunación. En Chile, se incorporó la vacunación de niños con vacuna conjugada el año 2011. En adultos mayores y con factores de riesgo, el PNI contempla la vacuna polisacárida Pn23 desde el año 2007. Sin embargo, globalmente la cobertura en adultos es extremadamente baja, menor a $15 \%$ en el $2016^{17}$, lo

Tabla 4. Mortalidad real y mortalidad teórica según índice CURB-65 en 68 casos de neumonía neumocócica bacteriémica, Hospital Puerto Montt 2010-2014

\begin{tabular}{|cccc|}
\hline CURB-65 & $\begin{array}{c}\text { Pacientes } \\
\mathbf{n}(\%)\end{array}$ & $\begin{array}{c}\text { Mortalidad real } \\
\mathbf{( \% )}\end{array}$ & $\begin{array}{c}\text { Mortalidad teórica } \\
\mathbf{( \% )}\end{array}$ \\
\hline 0 & $14(20,6)$ & 7,1 & 0,7 \\
1 & $14(20,6)$ & 14,3 & 2,1 \\
\hline 2 & $15(22,0)$ & 33,3 & 9,2 \\
\hline 3 & $15(22,0)$ & 46,7 & 14,5 \\
\hline 4 & $6(8,9)$ & 66,7 & 40,0 \\
\hline 5 & $4(5,8)$ & 100 & 57,0 \\
\hline
\end{tabular}


que se refleja en que sólo dos de nuestros pacientes habían recibido la vacuna antes de la hospitalización a pesar de tener indicación.

Con respecto al agente causal, obtuvimos el serotipo de $S$. pneumoniae en $96 \%$ (67/70) de las cepas. Cinco serotipos ( 1 , 7F, 3, 14 y 9V), causantes del cuadro en más de la mitad de los pacientes, están incluidos en las vacunas Pn23 y PV13. Otros siete serotipos $(9 \mathrm{~N}, 4,6 \mathrm{~B}, 11 \mathrm{~A}, 12 \mathrm{~F}, 19 \mathrm{~F}$ y $23 \mathrm{~F})$, responsables de 27\% de los casos (19/70) están incorporados en la vacuna Pn23. Esto significa que sólo alrededor de $10 \%$ de nuestros pacientes presentaron ENI no prevenible con vacuna. Una mejor cobertura tendría un impacto significativo en la morbilidad por S. pneumoniae.

El principal factor de virulencia de S. pneumoniae es el polisacárido capsular que permite clasificarlo en más de 90 serotipos. La cápsula es antifagocitaria y los anticuerpos opsonizantes de la respuesta inmunológica, confieren protección contra la ENI ${ }^{18,19}$. Nuestra casuística es pequeña y obtuvimos una importante dispersión de serotipos lo que dificulta valorizar la asociación entre serotipo y mortalidad. Sin embargo, hay indicios de diferencias asociadas al agente puesto que fallecieron 5/8 con serotipo 3 y 0/9 con serotipo 1 . Un meta análisis que incluyó 7 estudios de $\mathrm{ENI}^{20}$, concluye que serotipos como el 1 y $7 \mathrm{~F}$ tienen mayor capacidad invasora mientras otros se detectan principalmente en portación nasofaríngea (19F y 23F). Otro estudio de adultos con ENI, diseñado para correlacionar severidad con comorbilidad y serotipo $^{21}$, sugiere que algunos clones de los serotipos 1 y $7 \mathrm{~F}$ de alto potencial invasivo, se comportan como patógenos primarios y clones de serotipos como el $19 \mathrm{~F}$ y $23 \mathrm{~F}$ serían patógenos oportunistas, afectando pacientes con comorbilidad y produciendo enfermedad de mayor gravedad. Lamentablemente, no conocemos los serotipos de nuestra serie anterior ${ }^{5}$, pero en la actual, seis serotipos $(3,7 \mathrm{~F}, 14,9 \mathrm{~V}, 9 \mathrm{~N}, 11 \mathrm{~A})$ son causa de $71 \%$ de los fallecidos y los serotipos 3,14 y $9 \mathrm{~V}$, que en conjunto constituyen $30 \%$ de los casos, son responsables de casi la mitad de las muertes. Sin embargo, esto no alcanzó significación estadística, probablemente por el bajo número de pacientes, pero la tendencia se correlaciona con los hallazgos de los estudios mencionados y apoya que los serotipos o clones involucrados y no sólo el huésped, tienen impacto en la severidad de la ENI.

En varios países se reporta disminución de las ENI en adultos posterior a la introducción de la vacunación en niños ${ }^{22-24}$. Nuestros casos corresponden a un período próximo al inicio de la vacunación, por lo que este efecto aún no se puede evaluar. Tampoco se ha producido variación de los serotipos responsables de la ENI, fenómeno observado en otros países y la mayoría de los serotipos en nuestros pacientes, están incorporados en la vacuna que se administra a niños. En países que introdujeron la vacunación infantil previamente, se ha evidenciado un cambio de los serotipos de la ENI y su reemplazo por otros que ocuparían el nicho ecológico de sus predecesores ${ }^{25-27}$. En Chile, no hay evidencia que relacione la neumonía del adulto con cambio de serotipos, pero los datos del ISP muestran en los últimos años una variación global con disminución de los serotipos 1 y 14 y aumento de 3 y $19 \mathrm{~A}^{28}$. Este fenómeno podría ser un indicio del efecto de la vacunación en niños que, a diferencia de lo que ocurre en adultos, tiene una cobertura mayor a $90 \%{ }^{17}$. Disponer de los serotipos del estudio anterior ${ }^{5}$ nos habría permitido evaluar eventuales cambios locales.

En cuanto a los factores de la atención recibida durante la hospitalización, no identificamos aspectos que pudieran asociarse a mayor letalidad. El $100 \%$ de los casos recibió terapia antimicrobiana adecuada desde el ingreso. El retardo en el inicio del tratamiento específico puede modificar el pronóstico ${ }^{24,25}$, pero las limitaciones del diseño, no permitieron estimar el tiempo entre la aparición de síntomas, ingreso al HPM e inicio del tratamiento. Sin embargo, hay elementos que sugieren tardanza en el diagnóstico y uno de ellos es la gravedad al ingreso: $43 \%$ tenía infiltrados bilaterales, $16 \%$ compromiso pleural, $24 \%$ evolucionó con empiema o cavitación pulmonar y requirieron UCI y VM, 36\% y $29 \%$ respectivamente. También lo sugiere el exceso de mortalidad de los pacientes con CURB- $65 \geq 3(60 \%)$ y el que $83 \%$ de la mortalidad fue atribuible a la neumonía. Otras casuísticas chilenas de un período similar ${ }^{6,29}$ muestran menos infiltrados bilaterales al ingreso (30-37\%) pero similar compromiso pleural (10\%-25\%). Esto nos obligó a analizar factores adicionales que pudieran retrasar el diagnóstico. Estos podrían ser múltiples y algunos especulativos pero sabemos que $71,4 \%$ de los pacientes pertenecen a Fonasa A y B, que representa el nivel socioeconómico más bajo. Si agregamos la condición de ruralidad de $40 \%$ de la población atendida en el HPM y su- 
mamos limitaciones por la edad y comorbilidad, particularmente el alcoholismo, se configura un grupo que tiene menores posibilidades de acceder oportunamente a atención de salud y como consecuencia, presentan formas más graves y fallecen en mayor porcentaje. Las mismas razones explicarían que sólo dos pacientes estuvieran vacunados teniendo indicación. Los factores sociales, particularmente la pobreza, han sido poco estudiados en el pronóstico de la NAC, pero ya hay reportes que muestran que la NNB es más frecuente en población pobre ${ }^{30,31}$.

Creemos que futuros estudios que incluyan aspectos sociales, epidemiológicos y ambientales, no medidos habitualmente, podrían dar luces para identificar pacientes más vulnerables. Además, considerando que en los casos de mayor agresividad de nuestra población, el tratamiento específico no parece tener gran impacto, mejorar la cobertura de la vacuna, es decir la prevención, debiera tener un impacto significativo en la morbimortalidad por NNB.

\section{Referencias}

1. Riquelme R, Riquelme M. Rioseco ML, Gómez V, Gil $\mathrm{R}$, Torres A. Etiología y factores pronósticos de la neumonía adquirida en la comunidad en el adulto hospitalizado, Puerto Montt, Chile. Rev Med Chile 2006; 134: 597-605.

2. Bartlett JG, Mundy LM. Community-acquired pneumonia. N Engl J Med 1995; 33: 1618-24.

3. Welte T, Torres A, Nathwani D. Clinical and economic burden of community-acquired penumonia among adults in Europe. Thorax 2012; 67: 71-9.

4. Kalin M, Ortqvist A, Almela M, Aufwerver E, Dwyer R, Henriques B, et al. Prospective study of prognostic factors in community-acquired bacteremic pneumococcal disease in 5 countries. J Infect Dis 2000; 182: 840-7.

5. Rioseco ML, Riquelme R. Neumonia neumocócica bacteriémica en 45 adultos inmunocompetentes hospitalizados. Cuadro clínico y factores pronósticos. Rev Med Chile 2004; 132: 588-94.

6. Fica A, Bunster N, Aliaga F, Olivares F, Porte L, Braun $S$, et al. Bacteremic pneumococcal pneumonia: serotype, distribution, antimicrobal susceptibility, severity scores, risk factors and mortality in a single center in Chile. Braz J Infect Dis 2014; 18 (2): 115-23.

7. Amaro R, Liapikou A, Cilloniz C, Gabarrus A, Marco F, Sellares J, et al. Predictive and prognostic factors in pa- tients with blood cultura positive community-acquired pneumococcal pneumonia. Eur Respir J 2016; 48 (3): 797-807.

8. Capelastegui A, Zalacain R, Bilbao A, Egurrola M, Ruiz L, Quintana J, et al. Pneumococcal pneumonia: differences according to blood culture results. BMC Pulmonary Medicine 2014; 14: 128-35.

9. Musher D, Alexandraki I, Graviss E, Yanbeiy N, Eid S, Inderias L, et al. Bacteremic and nonbacteremic pneumonoccal pneumonia. A prospective study. Medicine 2000; 79: 210-21.

10. Fang GD, Fine M, Orloff J, Arisumi D, Yu VL, Singer $\mathrm{D}$, et al. New and emerging etiologies for community-acquired pneumonia with implications for therapy: A prospective multicenter study of 359 cases. Medicine 1990; 69: 307-16.

11. Charlson ME, Pompei P, Alez KL, MacKenzie CR. A new method of classifying prognostic comorbidity in longitudinal studies: development and validation. J Chronic Dis 1987; 40 (5): 373-83.

12. Lim WS, van der Eerden MM, Laing R, Boersma GW, Karalus N, Town GI, et al. Defining community acquired pneumonia severity on presentation to hospital: an international derivation and validation study. Thorax 2003; 58: 377-82.

13. Knaus W, Draper E, Wagner D, Draper E, Wagner D, Zimmerman J, et al. APACHE II: A severity of disease classification system. Crit Care Med 1985; 13: 818-28.

14. Clinical and Laboratory Standards Institute. M100 Performance Standards for Antimicrobial Susceptibility testing, 24th Edition.

15. Departamento de estadísticas e información en salud. Defunciones y mortalidad por causas. Disponible en http://www.deis.cl/defunciones-y-mortalidad-por-causas/ [Consultado el 15 de julio de 2017].

16. Superintendencia de Salud. Perfil epidemiológico del adulto mayor en Chile. Disponible en http://www. supersalud.gob.cl/documentacion/666/articles-4020_recurso_1.pdf [Consultado el 15 de julio de 2017].

17. González C. PNI MINSAL. Vacunas en adultos: Influenza y Neumococo. Disponible en http://web.minsal. cl/wp-content/uploads/2015/09/VC-Programa-Adulto-mayor-Vacunas-en-adultos-Influenza-y-Neumococcica.pdf [Consultado el 15 de julio de 2017].

18. Lindberg A. Glycoprotein conjúgate vaccines. Vaccine 1999; 17 (Suppl 2): 528-33.

19. Wood W, Smith M. The inhibition of surface phagocytosis by the capsular "slime layer" of pneumococcus type III. J Exp Med 1949; 90: 85-96.

20. Bueggemann A, Griffiths D, Meats E, Peto T, Crook D, Spratt B. Clonal relationships between invasive and 
carriage Streptococcus pneumoniae and serotype-and clone-specific differences in invasive disease potential. J Infect Dis 2003; 187: 785-96.

21. Sjöström K, Spindler C, Ortqvist A, Kalin M, Sandgren A, Kühlmann-Berenzon $S$, et al. Clonal ans capsular types decide whether pneumococci will act as a primary or opportunistic pathgen. Clin Infect Dis 2006; 42: 45159.

22. Muhammad R, Oza-Frank R, Zell E, Link-Gelles R, Narayan V, Schaffner W, et al. Epidemiology of invasive pneumococcal disease amomg high risk adults since the introduction of pneumococcal conjúgate vaccine for children. Clin Infect Dis 2013; 56: e59-e67.

23. Knol M, Wagenvoort G, Sanders E, Elberse K, Vlaminckx, Melker $\mathrm{H}$, et al. Invasive pneumococcal disease 3 years after introduction of 10 -valent pneumococcal conjuagate vacine, the Netherlands. Emerg Infect Dis 2015; 21: 2040-44.

24. Guevara M, Ezpeleta C, Gil-Setas A, Torroba L, Beristain X, Aguinaga A, et al. Reduced incidence of invasive pneumococcal disease after introduction of the 13.valent conjúgate vaccine in Navarre, Spain, 2001-2013. Vaccine 2014; 30: 2553-62.

25. Guevara M, Barricarte A, Gil-Setas A, García-Irure J, Beristain X, Torroba L, et al. Changing epidemiology of invasive pneumococcal disease following increased cove- rage with the heptavalent conjúgate vaccine in Navarre, Spain. Clin Microbiol Infect 2009; 11: 1013-9.

26. Richter S, Heilmann K, Dohrn C, Riahi F, Diekema D, Doern G. Pneumococcal serotypes before and after introduction of conjugate vaccines, United States, 19992011. Emerg Infect Dis 2013; 19: 1074-83.

27. Hausdorff W, Feikin D and Klugman K. Epidemiological differences among pneumococcal serotypes. Infection 2005; 5: 83-93.

28. Vigilancia de laboratorio de Streptococcus pneumoniae procedente de enfermedad invasora. Chile, 2010-2016. Disponible en http://www.ispch.cl/content/23910.

29. Saldías F, Viviani P, Pulgar D, Valenzuela F, Paredes S, Díaz O. Factores pronósticos, evolución y mortalidad en el adulto inmunocompetente hospitalizado por neumonía neumocócica adquirida en la comunidad. Rev Med Chile 2009; 137: 1545-52.

30. Burton D, Flannery B, Bennet N, Farley M, Gershman $\mathrm{K}$, Harrison L, et al. Socioeconomic and ratial/ethnic disparities in the incidence of bacteremic pneumonia among US adults. Am J Public Health 2010; 100: 190411.

31. Flory J, Joffe M, Fishman N, Edelstein P, Metlay J. Socioeconomic risk factors for bacteremic pneumococcal pneumonia in adults. Epidemiol Infect 2009; 137 (5): 717-26. 